\title{
CONTROL MODO DESLIZANTE APLICADO EN LA MALLA DE CORRIENTE PARA UNA APLICACIÓN DE UNA BASE-DSP PARA EL CONTROL DE POSICIÓN DE UN MOTOR DE INDUCCIÓN DE JAULA DE ARDILLA
}

\section{SLIDING MODEL CONTROL APPLIED IN CURRENT LOOP FOR A DSP- BASED POSITION CONTROL APPLIED TO SQUIRREL-CAGE INDUCTION MOTOR}

Eng. Wilman L. Bermeo, Dr. Antonio B. de Souza Jr., Dr. Tobias R. Fernandes N., MSc. Dalton A. Honório, Dr. Laurinda L. Nogueira dos Reis, Luiz H. S. C. Barreto.

Universidade Federal de Ceará.

Grupo de Automação e Robótica-GPAR, Departamento de Engenharia Elétrica. Caixa Postal 6001, 60.455-760 Campus do Pici, Fortaleza, CE, Brasil.

Tel.: (+55-85)-33669580, Fax: (+55-85)-33669574.

E-mail: \{wilmarbermeo, barbosa, tobias, dalton, laurinda, lbarreto $\} @$ dee.ufc.br

Resumen: Este articulo propone el control modo deslizante y el control por campo orientado usando referencia síncrona aplicada a un motor de inducción pequeño de jaula de ardilla utilizando modulación de largura de pulso espacio vectorial (SPWM) para el control de la posición y del torque, un procesador digital y el método de lazo cerrado con relé para evaluar los parámetros del controlador.

Palabras clave: Espacio Vectorial, Modo de Control Deslizante, Procesador de Señal Digital (DSP), Controlador PID, Método Modificado de Ziegler-Nichols.

\begin{abstract}
This paper proposes the sliding mode control and field-oriented control using synchronous reference applied to a small induction motor squirrel cage and using pulse length modulation vector space (SPWM) for controlling the position and torque, digital processor and closed loop method with relay to evaluate parameters of controller.
\end{abstract}

Keywords: Space Vector, Sliding Mode Control, Digital Signal Processor (DSP), PID Controllers, Modified Ziegler-Nichols Method.

\section{INTRODUCCIÓN}

Los modernos procesos industriales colocan exigencias rigorosas en unidades industriales por medio de la eficiencia, desempeño dinámico, características de funcionamiento flexibles, facilidad de diagnóstico y de comunicación con un computador central (gamboa et al, 2005). Estés juntamente con los desenvolvimientos en dispositivos de microelectrónica y de potencia llevaron a una tendencia firme para el control digital de drivers. Hay una gran variedad de aplicaciones, tales como las herramientas de las máquinas, elevadores (Osama y Abdul, 2008), robot (Leonhard, 1990), discos (Woodcock, 1979), etc. Donde el control rápido de la posición y del torque es fundamental. Estas aplicaciones son dominadas por drivers DC y no pueden ser operadas de forma satisfactoria para un motor de inducción con esquema constante Volt/Hertz (v/f). A lo largo de las dos últimas décadas el control vectorial de la máquina $\mathrm{AC}$ evoluciono, por medio de los cuales los motores AC y de inducción en particular, pueden ser controlados para obtener un desempeño dinámico comparable al que se es posible en una máquina DC. Estos controladores 
son llamados de controladores de vector porque controlan amplitud y fase de excitación AC. El control de los vectores de corriente y tensión resulta en el control de la orientación espacial de los campos electromagnéticos en la máquina lo cual lleva al término: orientación de campo. Normalmente, este término es reservado para los controladores que mantienen una orientación espacial de $90^{\circ}$ entre los componentes de campo crítico, por lo tanto, el control del ángulo de campo fue adoptado para los sistemas que se alejan de la orientación de $90^{\circ}$. La relación de deslizamiento es necesaria y suficiente condición para producir la orientación de campo (Novoty y Lipo, 1997). Las dificultades de usar la orientación de campo es el resultado de las incertezas debidas a la variación de los parámetros, saturación magnética, disturbios de carga y el no modelamiento de la dinámica. Para garantizar diversas estrategias de control robusto y diseño dinámico para accionamiento de motores de inducción tienen sido reportados en la literatura (Iwasaki y Matsui, 1993; Dunnigan et al., 1998). Un esquema en especial, sobre control deslizante, el cual tiene llamado la atención por su diseño simple, rápida respuesta dinámica, fácil implementación, robustez a variación de parámetros, y buena respuesta para disturbios de carga (Lin y Shiau, 1998; Chan y Wang, 1996).

SPWM se refiere a una técnica especial de determinación de la secuencia de conmutación de los tres transistores de potencia superiores en la fuente de tensión en un inversor trifásico. Tienen demostrado que generan menos distorsión harmónica en las tensiones de salida o corriente en los bobinados de la carga del motor (Trzynaklowsky y Andrzej, 1982). SVPWM proporciona la utilización más eficaz del BUS de tensión, en comparación con la técnica de modulación sinusoidal directa. Además, tiene una banda baja de armónicos que regulan el PWM y otros métodos de modulación sinusoidal, de otra forma optimiza los armónicos, impidiendo la conmutación desnecesaria, por lo tanto, menos perdidas de la conmutación. Estos tienen una abordaje diferente para la modulación PWM con base en la representación vectorial de las tensiones en el plano $d-q$ (Trzynaklowsky y Andrzej, 1982). El controlador usado tiene un PID, cuyos parámetros son calculados por el método de Ziegler-Nichols modificado, que tiene ventajas en comparación con otras técnicas de ajuste de parámetros PID, y se ha tornado un padrón industrial (Astrom et al., 1995) .Este articulo propone un control modo deslizante y campo orientado usando referencia síncrona para el control de la posición y del torque en un motor de jaula de ardilla usando SVPWM. Para la evaluación experimental de ambos algoritmos se usó un DSP TMS 320F2812 32 bits, que permite en tiempo real ver el algoritmo y el diseño costoefectivo de los controladores inteligentes del motor de inducción.

\section{MODELADO DINÁMICO DEL CAMPO ORIENTADO INDIRECTO PARA UN SERVO DRIVE MOTOR DE INDUCCIÓN}

El diagrama de bloque del experimento campo orientado indirectamente para un servo posicionador de motor de inducción es mostrado (Bose, 1986). El driver consiste en un servo motor de inducción, una comparación rampa inversor SVPWM actual, un mecanismo de orientación de campo, un traductor de coordenadas, una malla de control de velocidad interna y una malla de control de posición externa. El motor de inducción es usado en este sistema de drive en tres fases $\mathrm{Y}$ conectadas, 4-polos, $1 / 4 \mathrm{HP}, 60 \mathrm{~Hz}, 220 \mathrm{~V} 0.66 \mathrm{~A}$. las ecuaciones de estado de un motor de inducción en referencia síncrona rotativa puede ser escrita así (Bose, 1986):

$$
\frac{d}{d t}[A]=[B][A]+\frac{1}{\sigma L_{S}}[D]
$$

Donde,

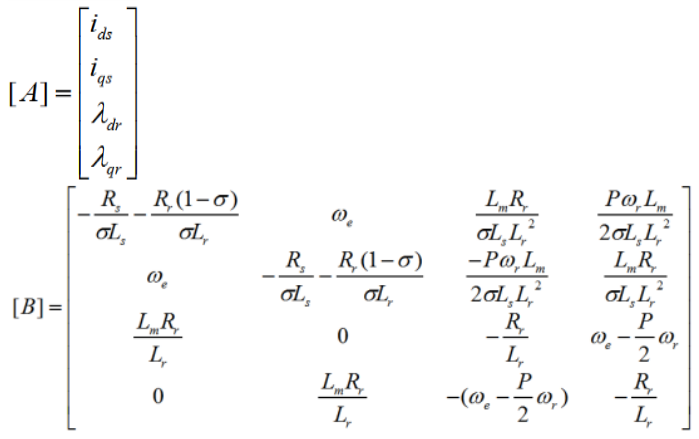

y

$$
\begin{aligned}
{[D]=\left[\begin{array}{l}
v_{d s} \\
v_{q s} \\
0 \\
0
\end{array}\right] } \\
T_{e}=\frac{3 P}{4} \frac{L_{m}}{L_{r}}\left(i_{q s} \lambda_{d r}-i_{d s} \lambda_{q r}\right) \\
\sigma=1-\frac{L_{m}{ }^{2}}{L_{s} L_{r}} \\
\lambda_{q r}=L_{m} i_{q s}+L_{r} i_{d r} \quad \lambda_{d r}=L_{m} i_{d s}+L_{r} i_{q r}
\end{aligned}
$$


$R_{s}:$ Resistencia del estator por fase.

$L_{s}$ : Inductancia de magnetización por fase del estator.

$R_{r}$ : Resistencia del rotor por fase referido al estator

$L_{r}$ : Inductancia de magnetización por fase del Rotor referido al estator.

$L_{m}$ : Inductancia de magnetización por fase.

$P$ : Número de polos.

$\omega_{e}$ : Velocidad angular eléctrica.

$\omega_{r}$ : Deslizamiento angular.

$V_{d s}$ : Tensión del estator eje-d.

$V_{q s}:$ Tensión del estator eje-q.

$i_{d s}$ : Corriente del estator eje-d.

$i_{q s}:$ Corriente del estator eje-q.

El modelo dinámico del motor de inducción y todo el sistema de accionamiento puede ser simplificado con el uso de control de campo orientado como muestra la figura 1 (Casadei et al., 2002). En un motor de inducción para tener una ideal orientación de campo, la disociación entre el eje-d es conseguida, y el total de la ligación del flujo del rotor es forzada a alinear en el eje- $d$ por lo tanto, el del flujo y la derivada es cero:

$$
\lambda_{q r}=0 \mathrm{y} \frac{d \lambda_{q r}}{d t}=0
$$

El vinculo del rotor se puede encontrar por medio de la tercera linea de la ecuación (1) y usando la ecuación (5) así:

$$
\lambda_{d r}=\frac{L_{m} i_{d s}}{1+s \frac{L_{r}}{R_{r}}}
$$

En comparación con la constante de tiempo del sistema mecánico, la constante de tiempo en la ecuación (6) se supone despreciable e $i_{d s}$ se establece constante $\left(i_{d s}=i^{*} d s\right)$ para la constante de flujo deseada del rotor. La ecuación queda en:

$$
\lambda_{d r}=L_{m} i_{d s}^{*}
$$

Para la ecuación 5 y 7 la ecuación de torque (2) se simplifica:

$$
T_{e}=\frac{3 P}{4} \frac{L_{m}^{2}}{L_{r}} i_{d s}^{*}
$$

Donde $i *_{q s}$ indica el comando de corriente de torque generado a partir del controlador de torque $G_{c}(\mathrm{~s})$.
Campo orientado indirectamente, el deslizamiento angular es necesario para calcular el vector unitario para las coordenadas de traslación. Usando la cuarta línea de la ecuación (1) y ecuación (5), la frecuencia de deslizamiento angular $\omega_{s l}$ puede ser estimada por:

$$
\omega_{s l}=\frac{L_{m} R_{r} i_{q s}^{*}}{L_{r} \lambda_{d r}}=\frac{R_{r} i_{q s}^{*}}{L_{r} i_{d s}^{*}}
$$

El torque generado, velocidad del rotor y posición angular del rotor $\theta_{r}$ son relacionadas por:

$$
\omega_{r}=s \theta_{r}=\frac{1 / J}{s+B / J}\left[T_{e}(s)-T_{L}(s)\right]
$$

Donde $B$ denota la frecuencia de amortecimiento viscoso y $J$ denota la constante de inercia.

\section{DISEÑO DE CONTROL DE CORRIENTE EN MODO DESLIZANTE}

Motor de inducción tiene una dinámica altamente no lineal. La principal dificultad en el control del driver IM (accionamiento del motor de inducción) surge de la multiplicidad de la no linealidad del torque electromagnético. Sin embargo, se desarrolla un algoritmo para el problema de control de corriente, con esto la velocidad y posición se pueden regular fácilmente con un controlador de malla externa. Para este fin, el esquema SMC es la primera aplicación de laso interno de control de corriente. Desde el punto de vista de modo de control deslizante, las trayectorias del sistema deben ser obligadas a abordar el colector es pecificado desde cualquier es tado inicial en el plano de estado (Utkin et al., 1999). A continuación, el comportamiento del sistema se rige por la dinámica del colector en el que se mantienen las trayectorias del sistema. A continuación, las trayectorias del sistema se moverán al origen del plano de estado a lo largo del colector dado.

Shiau (2001) tiene propuesto la ley de control que usa los parámetros de los controladores de posición y velocidad tomando en consideración el laso interno de control para el diseño del controlador de corriente modo deslizante, como muestra la fig. 1. La referencia de la señal de torque es reconocida como una variable de estado adicional al sistema global. 


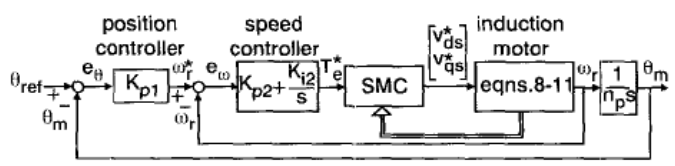

Fig. 1. Sistema global del driver de posición para motor de inducción (Shiau y Lin, 2001).

La propuesta de la ley de control es mostrada en la ecuación (8):

$$
\begin{aligned}
& {\left[\begin{array}{l}
v_{d s} \\
v_{q s}
\end{array}\right]=-L_{\sigma}\left(\left[\begin{array}{l}
\hat{f}_{1} \\
\hat{f}_{4}
\end{array}\right]+\left[\begin{array}{c}
0 \\
c_{2}
\end{array}\right] \dot{\theta_{r e f}} \cdot\left[\begin{array}{c}
0 \\
c_{3}
\end{array}\right] \theta_{r e f}\right)} \\
& -L_{\sigma}\left(\left[\begin{array}{cc}
q_{1} & 0 \\
0 & q_{2}+\mid c_{1} T_{L} I_{\max }
\end{array}\right]\left[\begin{array}{c}
\operatorname{sgn}\left(s_{1}\right) \\
\operatorname{sgn}\left(s_{2}\right)
\end{array}\right]+\left[\begin{array}{cc}
k_{1} & 0 \\
0 & k_{2}
\end{array}\right]\left[\begin{array}{l}
s_{1} \\
s_{2}
\end{array}\right]\right)
\end{aligned}
$$

Dónde:

$$
\begin{aligned}
& f_{1}=-\frac{1}{L_{\sigma}}\left(R_{s}+\frac{L_{m}^{2}}{\tau_{r} L_{r}}\right) i_{d s}+\frac{L_{m}}{\tau_{r}} \frac{i_{q s}^{2}}{\lambda_{d r}}+\omega_{r} i_{q s}+\frac{L_{m}}{\tau_{r} L_{\sigma} L_{r}} \lambda_{d r} \\
& L_{\sigma}=L_{s}-\frac{L_{m}^{2}}{L_{r}} \\
& \tau_{r}=\frac{L_{r}}{R_{r}} \\
& f_{4}=f_{2}-\frac{1}{k_{T} \lambda_{d r}} f_{3} \\
& f_{2}=\frac{1}{L_{\sigma}}\left(R_{s}+\frac{L_{m}^{2}}{\tau_{r} L_{r}}\right) i_{q s} \frac{L_{n} i_{q i_{d s}} i_{r s}+\omega_{d j} i_{d s}+\frac{L_{m}}{\lambda_{L} L_{r}} \lambda_{d r} \omega_{r}}{\tau_{3}=} \\
& f_{3} K_{p 2} k_{T} i_{q s} T_{L} \lambda_{d r}-\left(\frac{K_{p 2} K_{p 1}}{P / 2}+K_{i 2}\right) \omega_{r}-K_{p 1} K_{i 2} \theta_{m} \\
& c_{2}=-\frac{K_{p 2} K_{p 1}}{k_{T} \lambda_{d r}} \\
& c_{3}=-\frac{K_{p 1} K_{i 2}}{k_{T} \lambda_{d r}} \\
& q_{1}>\left|\hat{f}_{1}-f_{1}\right|+\eta_{1} \\
& q_{2}>\left|\hat{f}_{4}-f_{4}\right|+\eta_{2}
\end{aligned}
$$

$\eta_{1}, \eta_{2}, k_{1}, k_{2}>0$ y “^” denota parámetros de estimación. Los valores de $k_{1}, k_{2}$ son relacionados con el tiempo de la corriente alcanzada y $\eta_{1}, \eta_{2}$ con la atenuación de la vibración. También $q_{1}, q_{2}$ son usadas para lidiar con las variaciones de los parámetros eléctricos (Shiau, 2001).

Para la estabilidad del sistema global, el laso interno es despreciado, porque esta dinámica es mucho más rápida que la malla de posición y velocidad (Shiau, 2001). Así podemos reducir a la línea actual conseguida por SMC para el nuevo sistema mostrado en la figura 2.

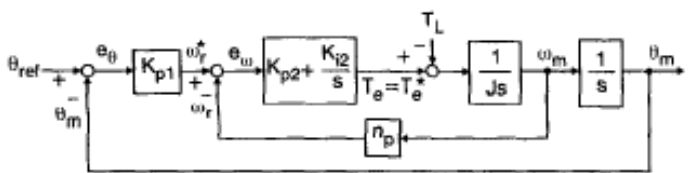

Fig. 2. Diagrama de bloques del sistema después del seguimiento de corriente. (Shiau y Lin, 2001).

Considerando la carga del torque como una perturbación del sistema, la función de transferencia de circuito cerrado a partir de la entrada de posición de referencia para la posición del eje en la figura 2 es dado por:

$\left.\frac{\theta_{r e f}}{\theta_{m}}\right|_{T_{L}=0}=\frac{K_{p 2} K_{p 1} s+K_{p 2} K_{i 2}}{J s^{3}+\frac{P}{2} K_{p 2} s^{2}+\left(K_{p 2} K_{p 1}+\frac{P}{2} K_{i 2}\right) s+K_{p 1} K_{i 2}}$

A pesar de que (Shiau y Lin 2001) sugiere usar alocación de polos se usará el método de ZieglerNichols modificado para tener una buena comparación.

\section{SIMULACIÓN Y RESULTADOS EXPERIMENTALES}

El servo motor de inducción usado en este sistema de accionamiento es trifásico Delta-conectado con 4 polos $1 / 4 \mathrm{CV}, 60 \mathrm{~Hz}, 380 / 220 \mathrm{~V} 0.66 / 1 \mathrm{~A}$. El fabricante da los parámetros necesarios para los teste de simulación. Aplicando el método de realimentación de relé para control interno (comando de controlador de torque (Bose, 1986)) para el vector de control en Simulink ${ }^{\circledR}$, el sistema muestra los resultados que pueden ser vistos en la figura 2. Con estos resultados, primero se usa la ecuación para encontrar el punto final (Astrom y Hagglund, 1995):

$G\left(i \omega_{a}\right)=-\frac{\pi a}{4 d}$

Dónde " $d$ " denota la amplitud del relé y " $a$ " es la amplitud de salida del sistema, el punto puede ser localizado en $r_{a}=0.0628$ y $\Phi_{a}=0$. Usando la sugerencia de (Pessen, 1954) y el método modificado de Ziegler - Nichols para realocar el punto final, los siguientes parámetros del controlador de torque son encontrados: $k_{p}=3.3616$ y $t_{i}=0.0044$ repitiendo el mismo método, pero con controlador exterior conectado (es decir, controlador de comando de posición) se obtienen los resultados mostrados en la figura 3 . 

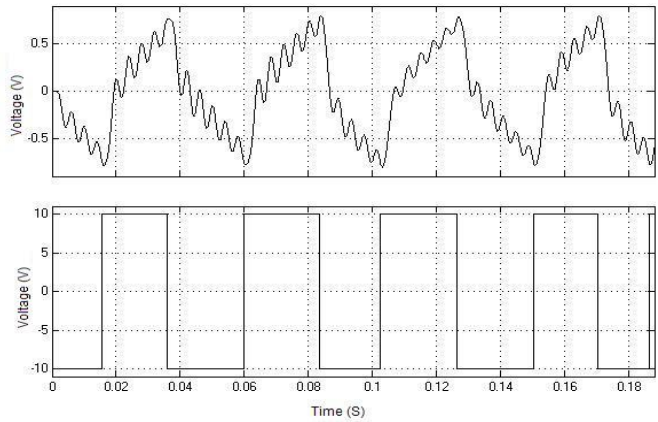

Fig. 3. Aplicación de relé lazo cerrado para torque.
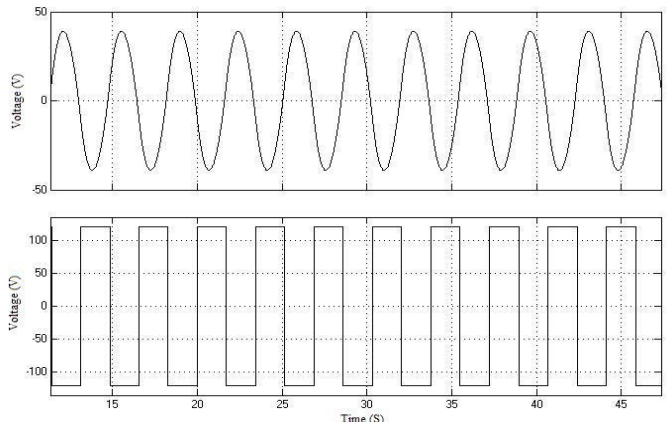

Fig. 4. Aplicación de relé lazo cerrado para controlador de posición.

El último punto en la curva de Nyquist para el sistema con controlador de torque PI es $r_{a}=0.2389$ y $\Phi_{a}=0$. Calculando los parámetros del PI usando el mismo criterio de Pessen $k_{p}=7.3432 \mathrm{y}$ $t_{i}=0.1591$. Aplicando estos parámetros de controlador para el esquema mostrado en la referencia (Bose, 1986) y figura 1, usando Simulink ${ }^{\circledR}$ para simular el sistema, se obtienen los resultados mostrados en la figura 5 .

La referencia de posición de 5 radianes fue aplicada para todo el sistema, y después 8 segundos después esta referencia fue cambiada para 2 radianes.

El error de estado estacionario para la posición es de $0.3 \%$. Esto se ve en la figura 5. El tiempo para estado estacionario, usando el criterio de $2 \%$, fue de 0.8 segundos. Como se puede ver en la figura 7 , aplicando el algoritmo de modo de control deslizante para el sistema permite a la posición del rotor ser más robusto y estar más cerca de la posición de referencia.

El torque electromagnético también es reducido, como se puede ver claramente en la figura 6 .

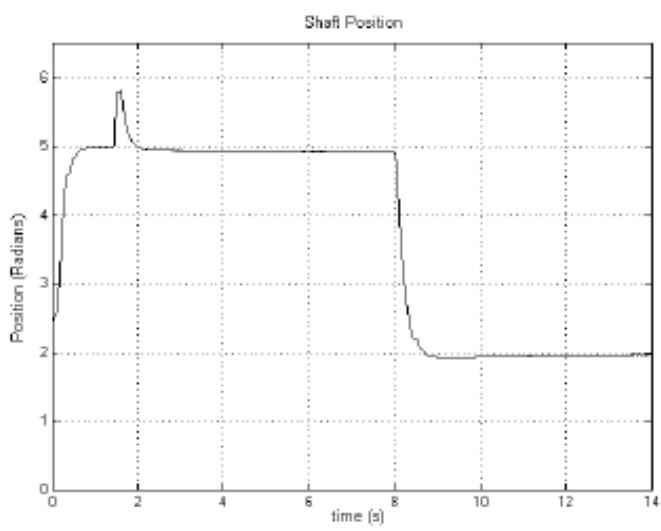

Fig. 5. Resultados de la posición del rotor de la simulación usando control de modo deslizante.

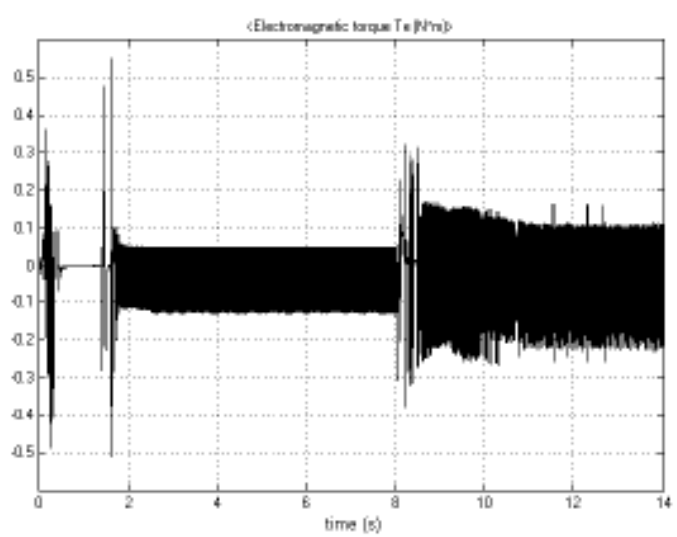

Fig. 6. Resultados del torque electromagnético de la simulación usando control de modo deslizante.

Los resultados experimentales para el modo de control deslizante son mostrados en la figura 7. El tiempo muerto, es demasiado pequeño por lo tanto es despreciado. El error de estado estacionario fue acerca de $0.7 \%$, similar a los resultados obtenidos en la simulación. El tiempo para estado estacionario, usando el criterio del $2 \%$, fue de 1.6 segundos. El torque es de $0.2 \mathrm{~N} / \mathrm{m}$, para el control modo des lizante.

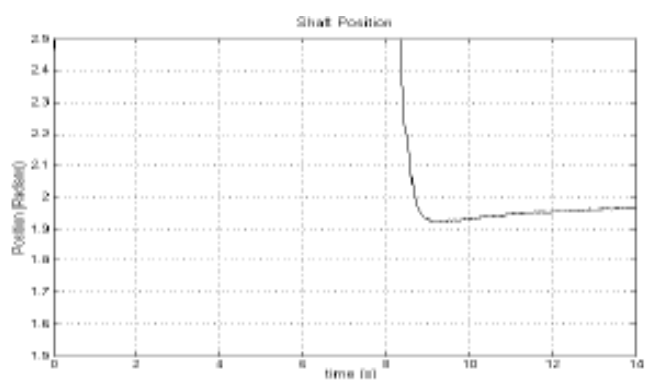

Fig. 7. Control modo deslizante para control de posición del rotor a partir de los resultados de la simulación. 


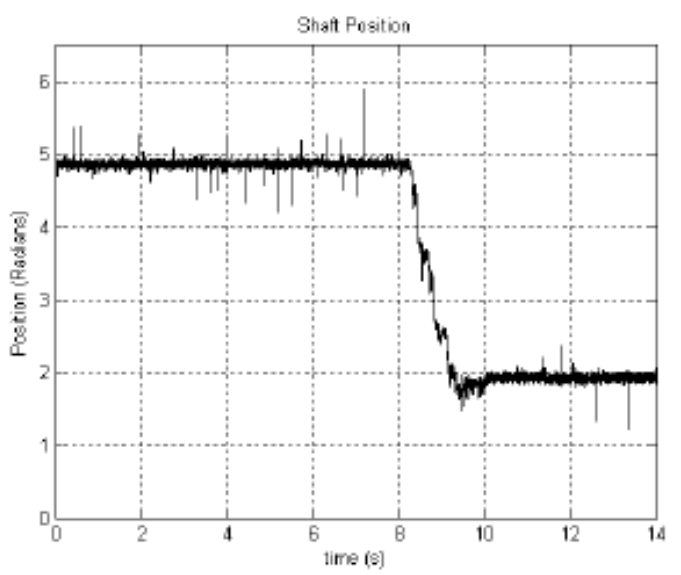

Fig. 8. Posición del rotor obtenida a partir de resultados experimentales usando control modo deslizante.

\section{CONCLUS IONES}

Este artículo propone un control modo deslizante, usando espacio vectorial, que es el modo de conmutación usado en TMS320F2812, ante la aplicación de los parámetros de control de la planta real. La DSP us a comando de control de tensión en vez de comando de corriente. En vez de esto el modo de control deslizante usa el comando de tensión. El método de Ziegler-Nichols fue usado para calcular los parámetros del PID en el control vectorial, mientras que la alocación de polos fue usada para el modo de control deslizante. El uso del modo de control deslizante incrementa el torque y reduce el tiempo de respuesta y el error de estado estacionario. Usando las ecuaciones propuestas en este artículo se puede construir un diagrama de bloques en Simulink ${ }^{\circledR}$, que genera el código para ser aplicado en la DSP. Los resultados de la simulación son próximos a los resultados experimentales, dejando que el ingeniero de diseño analice las respuestas del sistema sin necesidad de muchos testes experimentales.

\section{REFERENCIAS}

Astrom, K. y Hagglund, T. (1995). "PID Controllers: Theory, Desing and Tuning”. Instrument Society of America, $2^{\text {nd }}$ Edition.

Astrom, K., Lee, T. Than, K. Johansson, K. (1995). "Recent advances in relay feedback methodsa survey". Systems, Man and Cybernetics. 'Intelligent Systems for the 21st Century'. IEEE International Conference on Volume 3, 22-25 Oct. Page(s):2616 - 2621 vol.3.
Blaschke, F. (1971) "The Principle of Field Orientation - the Basis for the Transvector Control of Three-Phase Machines", Siemens Zeitschrift, Vol. 45, No. 10, pp. 757-760.

Bose, B.K. (1986) "Power Electronics and AC Drives". Prentice-Hall, 1st Edition.

Casadei, D; Profumo, F; Serra, G; Tani, A. (2002) "FOC and DTC: Two Viable Schemes for Induction Motor Torque Control". IEEE Transactions in Power Electronics, Vol. 17, No. 5.

Chan, C.C y Wang, H.Q. (1996). "New scheme of sliding-mode control for high performance induction motor drives", IEE Proc. Electr. Power Appl. Vol. 143, Issue1, pp. 177-185.

Chung-Yuen, W., Sei-Chan, K., Bose, B. K. (1992). Robust position control of induction motor using fuzzy logic control. IAS Annual Meeting, Vol. 1, pp. 472-482.

Gamboa, P. Faria, J. Margato, E. Palma, J. Ferreira, L. (2005) "Modern Technologies for experimental education in industrial electronics and electric drives", Power Electronics and Applications European Conference on, Dresden.

Holtz, J. (1998) "Pulse Width Modulation for Electronic Power Conversion". IEEE invited paper.

Honório, D. A., Diniz, E. C., de Souza, A. B., Almeida, O. M., Barreto, L. H. S. C. (2010). Comparis on between sliding model and vector control for a DSP-Based position control applied to squirrel-cage induction motor. IEEE/IAS International Conference on Industry Applications, pp. 1-6.

Iwasaki, M. y Matsui, N. (1993). "Robust speed control of IM with torque feedforward control". IEEE Transactions, IE40, (6). Pp553-560.

Novotny, D.W., Lipo, T.A. (1997). "Vector Control and Dynamics of AC Drives". Oxford Science Publications, 1st Edition.

Leonhard, W. (1990). "Trajectory control of a multi-axes robot with electrical servo drives", IEEE Control System Magazine, Vol. 10, Is sue 6, pp. 3-9.

Lin, J.L y Shiau, L.G. (1998). "PI speed control for an induction motor with inner sliding-mode torque control scheme". Proceedings of the automatic Control Conference:: of R.O.C. pp 515-519.

Osama, M. y Abdulazim, O. (2008). "implementation and performance analysis of an elevator electric motor drive system". $12^{\text {th }}$ 
International Middle-East Power System Conference, Aswan.

Pessen, B.W.(1954). "How to 'tune in' a three mode controller". Instrumentation. Second Quarter.

Shiau, L.G y Lin, J.L. (2001). "Staboçotu pf Sliding-mode current control for high performance induction motor position drives". IEE Proceedings in Electronic Power Applications, Vol.148, Is sue 1, pp. 69-75.

Raute, R., Caruana, C., Staines, C. S., Cilia, J., Sumner, M., Asher, G. M. (2010). Sensorles s control of induction machines at low and zero speed by using PWM harmonics for Rotor-Bar slottinh detection. IEEE International Conference on Transations Electronics, Vol. 46, pp. 1989-1998.
Trzynadlowski, Andrzej M. (1982). "The Field Orientation Principle in Control of Induction Motors". Springer Publishing, 1st Edition.

Utkin, V; Guldner, J; Shi, J. (1999). "Sliding Mode Control in Electromagnetical Systems". Taylor and Francis, $1^{\text {st }}$ Edition.

Wolovich, W.A. (1994). "Automatic Control Systems: Basic Analysis and Design". Saunders College Publishing.

$\mathrm{Yu}$, Z. (2001). "Space Vector PWM with TMS320C24x Using Hardware and Software Determined Switching Patterns". Application Report SPRA524, Texas Instruments. 